Mots. Les langages du politique

Regards sur le post-colonialisme linguistique

\title{
Quand la politique tire la langue. Le Vaffa Day du M5S (Movimento 5 Stelle)
}

When politics sticks its tongue out. The M5S' Vaffa Day

Cuando la política saca la lengua. El M5S' Vaffa Day

Laura Santone

\section{OpenEdition}

\section{Journals}

Édition électronique

URL : https://journals.openedition.org/mots/21810

DOI : $10.4000 /$ mots. 21810

ISSN : 1960-6001

Éditeur

ENS Éditions

Édition imprimée

Date de publication : 31 décembre 2014

Pagination : $87-103$

ISBN : 978-2-84788-544-6

ISSN : 0243-6450

\section{Référence électronique}

Laura Santone, "Quand la politique tire la langue. Le Vaffa Day du M5S (Movimento 5 Stelle) », Mots. Les langages du politique [En ligne], 106 | 2014, mis en ligne le 31 décembre 2016, consulté le 22 avril 2022. URL : http://journals.openedition.org/mots/21810 ; DOI : https://doi.org/10.4000/mots. 21810 


\section{Quand la politique tire la langue. Le Vaffa Day du M5S (Movimento 5 Stelle)}

Il était une fois Giuseppe Piero Grillo, mieux connu sous le nom de Beppe Grillo, artiste de cabaret devenu, dans les années quatre-vingt, comique satirique et acteur. En 1985, sur le plateau de Scemo di guerra de Dino Risi, il rencontre l'humoriste Michel Colucci, dit Coluche, ancien candidat à l'élection présidentielle française de 1981 sous le slogan : «Tous ensemble pour leur foutre au cul. »Cette rencontre, comme le rappellera plus tard Dino Risi interviewé par le Corriere della Sera ${ }^{1}$, est pour lui source d'inspiration. En 1986, à la suite d'un sketch où il attaque le Parti socialiste et le président du Conseil de l'époque, Bettino Craxi, Grillo est banni de la RAI, la télévision étatique italienne. Il commence alors à faire des tournées avec des spectacles qui seront diffusés par la Radiotélévision suisse italienne (RTSI) et par la chaîne française Canal Plus. Les thèmes abordés sont l'environnement et la politique, et le schéma narratif adopté est toujours le même : les "gentils», c'est-à-dire les Italiens, doivent vaincre les «méchants », c'est-à-dire les hommes politiques, notamment Berlusconi. Le show-man commence ainsi à attirer les foules en les galvanisant avec son énergie sulfureuse, et la presse ne tarde pas à parler de lui comme du «Coluche à l'italienne $»^{2}$. Dans ses monologues truffés de slogans, métaphores et hyperboles, le «dire» va de pair avec le «rire»: Grillo cherche la foule, la touche, la dynamise par son humour meurtrier, si bien que le ludique contamine le politique, le comique recouvre le sérieux, et l'entertainment se confond avec l'infotainment (Brants, 2003, p.135-166).

Alors qu'en 2000, il diabolisait les ordinateurs et les nouvelles technologies³, Grillo se convertit au Web dans les années suivantes. Il a rencontré entretemps celui qui va devenir son alter ego, Roberto Casaleggio, auteur de /l Web

1. Un passage de cette interview est cité par Giuliano Santoro, auteur de Un Grillo qualunque. II Movimento 5 Stelle e il populismo digitale nella crisi dei partiti italiani, 2012, p. 18.

2. Ainsi quelques titres : «Beppe Grillo, le Coluche italien qui bouleverse la politique » (MYTF1 News, 25 mai 2012); «L'ispiratore di Grillo? Coluche comico e capopolo » (L'Unità, 18 mai 2012); «Beppe Grillo, pari réussi pour le Coluche italien anti-système » (MYTF1 News, 26 février 2013); «Beppe Grillo, entre Coluche et Che Guevara » (LeFigaro.it, 22 février 2013).

3. Dans son spectacle Time Out. Voir Santoro, 2012, p. 33-34. 
è morto, viva il Web et Web ergo sum4, que la presse qualifie de "gourou télématique » du Movimento 5 Stelle ou M5S (Mouvement 5 Étoiles) 5.

26 janvier 2005 : Grillo débarque sur la toile, avec son blog beppegrillo.it, lancé en concomitance avec son spectacle payant Beppegrillo.it. Grillo devient l'homme du blog6. Son site s'impose comme l'un des plus visités d'Italie7 et l'hebdomadaire The Observer 8 le classe parmi les dix blogs les plus influents au monde. Fleurissent, en même temps, les MeetUp, un réseau de forums en ligne canalisant en différents groupes les «fidèles» de Grillo en fonction de leurs intérêts et de leur localisation géographique. Grillo devient ainsi le showman/chaman du Web, dont la formule magique par excellence sera «Vaffanculo» - «Va te faire foutre» -, matrice de la formule Vaffa Day ${ }^{9}$.

Appuyée sur un corpus qui couvre différents moments du M5S de 2007 à 2014, notre analyse vise à cerner, dans un premier temps, les éléments dont relève la formule Vaffa Day et, dans un deuxième temps, à repérer la texture des réénonciations, paraphrases, appellations et néologismes qui se sont propagés autour de cette formule dans l'espace public de l'interdiscours.

\section{L’homme du Web, la politique du Vaffa}

Dans Tutto il Grillo che conta (Grillo, 2006) anthologie où Grillo réunit douze ans de monologues, on trouve un extrait de Beppegrillo.it, tournée de 70 étapes dont la chaîne suisse RTSI transmet les trois soirées tenues en mars 2005 à Milan, devant un public dans lequel on peut reconnaître, entre autres, le prix Nobel Dario Fo ${ }^{10}$. Des 7500 mots qui composent l'extrait, deux occurrences résonnent en coréférence : cazzo («bite»), répété seize fois, et culo («cul»),

4. Publié en 2001 aux Éditions Pro-Sources, le livre présente le Web comme le lieu idéal d'une démocratie directe où les rapports sociaux se dégagent directement du pouvoir démiurgique de la Toile.

5. Emblématique, le titre d'un article publié par The Guardian le 3 janvier 2013 : «Italy's web guru tastes power as new political movement goes viral».

6. Qui porte le logo de Casaleggio \& Associati.

7. On trouve à ce propos, dans Un grillo qualunque (p.61), ce témoignage significatif : «Le matin, dès que j'arrive dans mon bureau, je m'assieds à ma table de travail, j'allume mon ordinateur et je me connecte au site de Grillo. J'ai été frappé par sa rage lors de son spectacle sur le vif. »

8. The Observer, 9 mars 2008.

9. Signalons que vaffa, abréviation de «vaffanculo », tout comme la locution française «va te faire foutre », outre qu'elle recouvre une sphère sémantique sexuelle, désigne par extension une expression de rejet, une formule de congé brutal relevant d'une rhétorique injurieuse et vulgaire. Il s'agit donc, comme dirait Christian Plantin, d'une définition ad hoc, «taillée à la mesure de la cible», qui exprime d'emblée une prise de position «vis-à-vis de l’objet défini » (Plantin, 1996, p. 53-54).

10. À l'instar de Coluche qui, à l'époque de sa campagne présidentielle, avait pu compter sur le soutien de certains intellectuels dont Pierre Bourdieu, Félix Guattari et Gilles Deleuze, et de vedettes du show-business, Grillo peut se vanter d'appuis tout aussi prestigieux ou notoires, dont, notamment, ceux du prix Nobel Dario Fo ou du chanteur pop Ligabue. 
répété treize fois. Ces mots résonnent également comme des «mots-virus» voués à se répliquer par contamination dans le canevas de tous les monologues de Grillo et, de là, à s'infiltrer dans les «cellules» du futur M5S : ils affectent d'une façon symptomatique le langage et les rapports mêmes des grillini $^{11}$ entre eux. En témoignent certains posts de militants au lendemain de ses spectacles:

Un capolavoro. Grillo come al solito ci spiega come ci pigliano per il culo i potenti e i capi di impresa. Ma che dobbiamo fare?

Fabrizio (7 mai 2006) ${ }^{12}$

Sono stanco di essere preso per il culo! Quando mi sento dire che votare è un dovere civico! Ma dove?

Marco Marconi (2 septembre 2005, 11 h 22) ${ }^{13}$

CIAO BEPPE... OGNI VOLTA CHE TI ASCOLTO, COME IERI A MILANO, MI SENTO PIÙ INFORMATO PIÙ FORTE PIÙ CARICO PER PROSEGUIRE LA MIA VITA E PER FARMI INCULARE OGNI GIORNO UN PO'MENO.

Carlo Oggioni (11 mars 2005, 13 h 52)14

L'effet de cumul anaphorique d'expressions obscènes qui en ressort - ci pigliano peril culo, essere preso peril culo, farmi inculare - met déjà en scène le paradigme d'une rhétorique virulente, où le médium - le Web -, en favorisant la polémique, l'emporte sur toute forme de débat en tant que forme d'interaction dialectique orientée par une question. Ce qui explique la devise de Grillo : «Ce n'est pas moi, c'est le médium»; et encore, sur la page inaugurale de son blog : "Je suis un partisan de la troisième guerre mondiale, celle de l'information. » La logique de réseau sous-jacente à cette déclaration de guerre joue la carte du «parlervrai » au sens d'un parler grossier qui n'est ni de droite, ni de gauche, ni du centre; il s'agit, à y regarder de près, d'une langue poussée de façon quasi mécanique à la limite de l'audible - de l’inouï - ; une sorte de novlangue volontairement habillée d’obscénités, qui «semble transformer

11. Grillini est le dérivé adjectival qui désigne les adeptes de Grillo. Les médias français le traduisent par «grillons» (ainsi, par exemple, LeMonde.fr, 4 mars 2013) ou, dans un emploi un peu détourné, par «grillistes», appellation forgée par le spécialiste de la vie politique italienne Marc Lazar (Le Nouvel Observateur, 22 février 2013). Il vaut la peine de signaler que le mot grillini fait partie, avec «tsunami », des entrées proposées par Lorenzo Pregliasco dans /l Crollo. Dizionario semiserio delle 101 parole che hanno fatto e disfatto la Seconda Repubblica (2013).

12. «Un chef-d'œuvre ! Grillo comme toujours nous explique comment les tout-puissants et les chefs d'entreprise se foutent de nous. Mais qu'est-ce qu'on doit faire? » (Notre traduction, comme c'est le cas pour toutes les citations italiennes). Dans les posts repérés, c'est nous qui soulignons les expressions directement apparentées, au niveau sémantique, à Vaffa Day.

13. "J'en ai marre qu'on se foute de moi! Quand on me dit que voter, c'est un devoir civique! Mais où?» http://www.beppegrillo.it/2005/01/milano.html. Dernière consultation des sites mentionnés dans cet article : 3 octobre 2014 .

14. «Salut Beppe... À chaque fois que je t'écoute, comme hier à Milan, je me sens plus branché, plus fort, plus déterminé à continuer à vivre et à me faire foutre chaque jour un peu moins. » Le choix des majuscules est celui du blogueur. 
celui qui la pratique en machine ${ }^{15}$ et que Grillo manipule sciemment en la déclinant - et en la pathémisant - sur tous les tons de la colère et de l'impudence. Son blog et les réseaux sociaux associés - Facebook, Twitter, les MeetUps - deviennent ainsi l'espace agonal d'un dissensus exaspéré, branché sur la haine, sur l'invective, et poussé à l'extrême par le biais d'une forme de gradation lexicale dans laquelle mots grossiers, choquants, insultants s'imbriquent entre eux tout en tissant la politique - et la rhétorique - du vaffa. Version bémolisée de "vaffanculo», que les dictionnaires répertorient à l'unanimité comme «expression injurieuse adressée à ceux qui nous dérangent, qui nous ennuient, pour qu'ils s'en aillent ${ }^{16}$, cette abréviation fait office de ciment identitaire parmi les adeptes de Grillo. Elle se configure comme le «logo » d'une stratégie de disqualification ad personam des adversaires ${ }^{17}$, frontalement attaqués et ridiculisés, comme on le verra, sous l'angle des passions et par la force du clamor en tant que dispositif d'alimentation de pathos négatif.

De la place virtuelle du Web à la place publique, le vaffa, symbole trivial du politiquement incorrect, est l'élément catalyseur de la masse et de ses affects et se révèle à la base d'un traitement phatique et spectaculaire du dissensus qui tend à aplatir toute forme d'argumentation horizontale. Car ce vaffa, apparemment tourné vers la masse allocutaire, résonne verticalement sous les traits, habilement occultés par l'ingrédient comique, d'un mot d'ordre tranchant net toute forme d'argumentation et toute ligne de partage communicationnelle entre Grillo, la multitude de ses fidèles et la classe politique italienne ${ }^{18}$.

Le 8 septembre 2007, le blog beppegrillo.it annonce la journée de « la svolta del calcio in culo» («le tournant du coup de pied au cul»), aube d'une «nouvelle Renaissance» pour recommencer à "sourire à la vie et être heureux»19. Le rendez-vous est à Bologne, Piazza Grande, où, devant 50000 personnes, Grillo ouvre le premier Vaffa Day. En hurlant à tue-tête «vaffanculo » et en haranguant la foule au son d'injures et d'anathèmes, il s'en prend à la classe politique italienne, désignée comme la «caste corrompue ». C'est à cette occasion que Michele Serra forge le mot grillismo, néologisme allant de pair avec grillini

15. Décrite par Orwell dans 1984, la novlangue est la langue du système totalitaire par excellence et se caractérise essentiellement par son utilisation mécanique. Mais, comme le souligne Michaël Oustinoff, « la novlangue n'est que l'aboutissement, sur le mode fictionnel, d'une réflexion profonde sur la langue en général et sur son utilisation dans l'espace public en particulier - et pas seulement dans les régimes totalitaires. Il est donc facile, notamment, de parler de novlangue en démocratie» (Oustinoff, 2010, p. 17). Ce qui semble être ici exactement le cas.

16. Nous traduisons ici la définition donnée par le dictionnaire Treccani, consultable en ligne à l'adresse: http://www.treccani.it/vocabolario/tag/vaffanculo/.

17. Comme le remarque Ruth Amossy, il est préférable de parler d'attaque ad personam, plutôt que d'attaque ad hominem, «quand elle confine à l'insulte» (Amossy, 2006, p. 143).

18. Ce qui explique, entre autres, la pratique d'«épuration » exercée par Grillo à l'encontre des militants qui transgressent les règles du $\mathrm{M}_{5} \mathrm{~S}$, notamment l'interdiction de participer aux débats télévisuels, ainsi que son refus a priori de tisser des alliances politiques autant avec la gauche qu'avec la droite.

19. http://www.beppegrillo.it/2007/o9/piazza_maggiore.html. 
et confirmant la progression du mouvement. La journée de Bologne, transmise en direct par Eco TV, recueille en quelques heures plus de 33000 signatures d'adhésion et présente immédiatement les contours d'un «événement» (Moirand, 2007). Ses échos se font entendre sur les cinq continents, et des blogs du monde entier décrivent la journée. Des médias à la blogosphère, on assiste à la scénarisation médiatique du Vaffa Day, que la traduction dans les différentes langues contribue à amplifier et à restituer avec une coloration sémantique - et connotative - supplémentaire. D'une traduction littérale restituant le Vaffa Day comme «la journée du va-te-faire-foutre ${ }^{20}$ ou «la journée du vate-faire-enculer » ${ }^{21}$, jusqu'aux traductions euphémisées telles que «la journée du va te faire... », la journée «to tell politicians " ${ }^{\star \star *}$ off" ", ou encore la «V-Day Celebration ${ }^{22}$, qui voilent l'injure sous des points de suspension, des astérisques ou par une désignation périphrastique, le syntagme révèle d'un côté son prédicat de réalité, soit le " "point de vue" axiologiquement inscrit dans la catégorisation ou la [dis]qualification » (Siblot, 2007, p. 38), et de l'autre s'installe dans le hic et nunc du discours sous forme de postulat idéologique. Vaffa Day accède ainsi au rang de formule au sens qu'en donne le Dictionnaire d'analyse du discours de Patrick Charaudeau et Dominique Maigueneau :

Dans son emploi spécialisé, le terme formule désigne une expression lexicale, le plus souvent un syntagme nominal ou une collocation à caractère néologique, qui renvoie à une notion ayant joué sur le plan idéologique un rôle fondateur et actif dans une situation historique. (Charaudeau, Maingueneau, 2002, p. 274)

«Rôle fondateur et actif» où joue, bien évidemment, la mise en relation du locuteur avec un procès de référenciation, ce qui fait de la formule Vaffa Day une séquence participant à la fois du discours et de l'interdiscours, à mi-chemin entre le plan de la signification littérale et le plan de la doxa. Elle devient, pour le dire avec Alice Krieg-Planque, « une séquence verbale, formellement repérable et relativement stable du point de vue de la description linguistique, [qui] se met à fonctionner dans les discours produits dans l'espace public comme une séquence conjointement partagée et problématique» (Krieg-Planque, 2003, p.14).

La formule Vaffa Day, en d'autres termes, se met à circuler dans le corps social en se propageant comme un objet de langue "partagé», qui s'inscrit dans le savoir collectif des locuteurs. Simultanément abrégée - ou reformulée - en

20. Comme le traduisent, par exemple, Libération du 7 septembre 2007 , Le Monde diplomatique d'avril 2008, Le Monde du 21 février 2013, Presseurop.eu du 7 février 2014.

21. Voir, surhttp://la-brochure.over-blog.com/article-le-livre-fabuleux-de-federico-mello-116146141. html, le compte rendu du livre de Federico Mello, Il lato oscuro delle stelle (2013).

22. Dans l'ensemble d'expressions euphémisées que nous avons repérées pour la traduction de Vaffa Day, il nous semble intéressant de mentionner celle de l'International Herald Tribune (13 septembre 2007) : "Beppe Grillo is the man behind V-Day (the Vstands for a very rude Italian expletive). » Alors que BBC News Europe (26 février 2013), en brossant le profil de Grillo, écrit : "In 2007 he organised "V-day" - the Vstands for a well-known Italian obscenity... ". 
$V$-day, elle fait très rapidement son entrée « encyclopédique » dans les pages de la plateforme italienne de la communauté numérique Wikipédia. Ainsi l'incipit :

II V-day (abbreviazione di Vaffanculo-day che riprende nel simbolo la V di V per Vendetta) è stata un'iniziativa politica italiana promossa dal comico Beppe Grillo. ${ }^{23}$

L'entrée est accompagnée de treize notes, dont la première renvoie à une glose que Grillo postait sur son blog le 14 juin 2007 :

L'estate sarà molto calda. Poi verrà settembre e il Vaffanculo-day o V-day. Una via di mezzo tra il D-Day dello sbarco in Normandia e V come Vendetta. Si terrà sabato 8 settembre nelle piazze d'Italia, per ricordare che dal 1943 non è cambiato niente. leri, il re in fuga e la nazione allo sbando, oggi politici blindati nei palazzi immersi in problemi 'culturali'. ${ }^{24}$

La formule trouve en même temps une cristallisation supplémentaire sur la page de la plateforme anglaise de la communauté Wikipédia. Cette entrée, beaucoup plus synthétique, trace en quelques paragraphes le paradigme dans lequel se situe le «V-day» de Beppe Grillo. Plus précisément :

- $V$-Day (movement), the global movement to end violence against women and girls;

- V-Day, a political campaign in Italy organized by Beppe Grillo;

$[\ldots]$

- Victory Day, the day an operation successfully concludes, see military designation of days and hours;

[...]

- V-E Day or Victory in Europe Day, the public holiday celebrated in May 1945 to mark the end of World War II in Europe;

- V-J Day or Victory over Japan Day, the day on which the surrender of Japan occurred, effectively ending World War II, and subsequent anniversaries of that event. 25

Paradigme qui dessine, il est aisé de le constater, le cadre mémoriel de la formule, son rapport à des dires et des faits emmagasinés en mémoire. Mémoire de discours antérieurs, stockés au fil du temps, que Grillo de son côté convoque en filant dans une chaîne d'associations apparentées la formule Vaffa Day.

23. «Le V-day (abréviation de Va-te-faire foutre qui reprend dans son symbole le V de V pour Vengeance) est une initiative politique italienne promue par le comique Beppe Grillo. » Voir http:// it.wikipedia.org/wiki/V-Day. Dans cette citation comme dans la suivante, les mots en gras figurent comme tels dans le texte original.

24. "L'été sera très chaud. Et après viendra septembre et le Vaffanculo-day ou V-day. À mi-chemin entre le D-Day du débarquement en Normandie et $V$ comme Vengeance. Il se déroulera samedi 8 septembre sur les places d'Italie, pour rappeler que depuis 1943 rien n'a changé. Hier, le roi en fuite et la nation en déroute, aujourd'hui, les politiciens blindés et submergés par des problèmes “culturels” ". Voir http://www.beppegrillo.it/2007/06/vaffanculoday.html.

25. http://en.wikipedia.org/wiki/V_Day. 


\section{La formule filée}

Une remarque préliminaire s'impose à ce stade de notre analyse sur l'utilisation de l'épithète filée dont nous allons nous servir relativement au fonctionnement de la formule "Vaffa Day». Ce qualificatif est redevable de la notion de métaphore filée que Michel Riffaterre applique à certains effets stylistiques de l'écriture automatique dans sa célèbre étude "La métaphore filée dans la poésie surréaliste», où, avant d'analyser les saillances qu'elle inscrit dans la construction verbale, il observe : «Or, par sa nature même, la métaphore filée constitue typiquement un code spécial, puisque les images qui la composent n'ont de sens, individuellement comme en groupe, qu'en fonction de la première d'entre elles» (Riffaterre, 1979, p. 217). Axiome sur lequel il greffe la définition suivante : "Ce qu'on appelle métaphore filée est en fait une série de métaphores reliées les unes aux autres par la syntaxe [...] et par le sens : chacune exprime un aspect particulier d'un tout, chose ou concept, que représente la première métaphore de la série » (ibid., p. 218). D’où ce corollaire : «Qui plus est, la création de ce code spécial a pour corollaire la destruction du code linguistique» (ibid., p. 225).

En partant de cette perspective, il va de soi que si nous appliquons la notion de «métaphore filée» à la formule, on devra parler, nécessairement, de «formule primaire». Il s'ensuit qu'on devra lui reconnaître, en tant que telle, la même propriété que Riffaterre dégage de «la première métaphore de la série», à savoir celle d'être, en tant que matrice, « véhicule » d'autres mots identiques, apparentés aux - ou dérivés des - mots utilisés dans un passage précédent. Propriété grâce à laquelle la métaphore filée se révèle susceptible de s'étendre à un ensemble de phrases en utilisant différents signifiants qu'elle relie en un réseau sémantiquement cohérent. Mais, qui plus est, cette propriété est loin d'épuiser sa fertilité, car la métaphore filée, comme le démontre de façon convaincante Philippe Dubois (1975, p. 209), mobilise en même temps un développement conceptuel de nature isotopique qui rattacherait la métaphore primaire à un parcours associatif complémentaire mettant en valeur, au fil du texte, la notion de bi-isotopie, les systèmes associatifs engendrés étant essentiellement au nombre de deux et se manifestant conjointement. Ce qui ne va pas sans renforcer, au niveau lexico-syntactique, son caractère «spécial» et sa vocation à la « destruction » du code linguistique.

Or, pour revenir à notre analyse, les deux notions de «code spécial» et de «destruction » évoquées par Riffaterre semblent bien s'adapter au « lexique des temps féroces ${ }^{26}$ que la formule Vaffa Day synthétise, dans la mesure où elle

26. D'après l'expression utilisée par Ilvo Diamanti, professeur de Sciences politiques à l'université d'Urbino. Alors que Stefano Bartezzaghi, dans son article «L'era del politicamente scorretto », paru dans 
semble résumer, tout en les démultipliant dans la texture de la Toile, les mots et les lieux d'une agressivité langagière sans précédent, intrinsèque à la rhétorique et à la politique que le vaffa est censé filer. Mais procédons dans l'ordre.

23 février 2008 : le MeetUp napolitain «Amici di Beppe Grillo » organise le Muday ou Munnezza day, également décliné comme "giornata del rifiuto ${ }^{27}$. De la Piazza Dante à la Piazza du Plébiscite, la protestation contre les tonnes de déchets qui envahissent les rues de Naples joue délibérément sur le double sens de rifiuto : derrière la notion d'ordure, le mot évoque délibérément sa signification première de « rejet », plus précisément le rejet de la classe politique italienne et de l'administration de la région Campanie, accusées de connivence avec la camorra. La «teneur » de la manifestation est conforme au schéma bolognais du Vaffa Day, dont le $\mathrm{Mu}$-day reprend manifestement le moule morphologique tout en opérant un déplacement avec le préfixe «Mu». Sorte de préfixe «animal», résonnant manifestement en écho avec le meuglement des vaches, ce «Mu » se veut être exactement un prolongement - ou une projection - du mot d'ordre vaffanculo, sémantiquement retrempé en raison de son élargissement référentiel. Le modus operandi est toujours le même : monter sur la scène et déclencher les passions en utilisant la blague comme arme de la polémique la plus exacerbée, dans un jeu de discrédit où le mot vaffanculo dynamite le langage et la politique tout en tenant lieu d'argumentation. La blogosphère, de son côté, tisse une autre association : le Mu-day ou Munnezza-day ("Journées des ordures»), déjà " giornata del rifiuto» ("Journée du refus»), est reformulée comme "giornata della vergogna ${ }^{28}$ («Journée de la honte»). Une blogueuse écrit ainsi :

Se il V-day aveva suscitato ilarità allora è lecito chiedersi le reazioni sulla nuova iniziativa dal singolare titolo Mu-Day o, più precisamente, Munnezza day [...] Credo sia il caso di farsi sentire anche dalla blogosfera. Facit'accussì : copiate 'sto testo MU-DAY. METTITEVE SCUORNO (traduzione : VERGOGNA). 29

De même, le titre en une de la page de Naples du quotidien en ligne Repubblica.it: «Rifiuti, nasce la giornata della vergogna, ecco il Munnezza day» 30 .

La Repubblica.it du 10 février 2008, a parlé de «parole-ringhio » [mots-grognement]. Voir aussi, de Bartezzaghi, « Le parole-ringhio che rompono i nostri tabù », La Repubblica.it, 21 août 2010.

27. Le mot dialectal munnezza traduit en napolitain immondizia, soit « déchets ", "ordures».

28. Lors de sa deuxième édition, le 9 avril 2011.

29. «Si le V-Day avait déclenché l'hilarité générale, il vaudrait mieux s’interroger sur les réactions que va susciter la nouvelle initiative au titre très singulier : le Mu-Day ou, plus précisément, Munnezza Day [...] Je crois qu'on doit se faire entendre aussi par la blogosphère. Faites comm'ça, copiez c'texte : / Mu-Day. Mettiteve scuorno (traduction : honte à vous). » Voir http://logga.me/ iotessy/2008/01/31/23-febbraio-il-munnezza-day/. C'est la blogueuse elle-même qui traduit en italien la locution napolitaine «Mettiteve scuorno» [honte à vous-mêmes; en gras dans le texte original]. À noter, également, le recours à d'autres lexèmes faisant partie du dialecte napolitain, tels que «facit' accussì » [faites ainsi] et «copiate 'sto testo » [copiez ce texte].

30. http://napoli.repubblica.it/cronaca/2011/04/06/. En français : « Déchets, arrive la journée de la honte, la journée des ordures». 
La formule, ainsi, se fraye un chemin dans l'espace public du discours et, avec le deuxième Vaffa Day, qui se déroule à Turin le 25 avril 2008, deux mois seulement après le Munnezza day / Mu-day, elle se stabilise comme référent social dans l'exacte acception indiquée par Krieg-Planque (2006) : "Ce qui caractérise la formule comme référent social, ce n'est pas qu'elle fasse l'unanimité (puisque bien au contraire elle ne fait pas consensus), mais c'est qu'elle représente, à un moment donné, un passage obligé.»

Elle devient ainsi, chemin faisant, le présupposé nécessaire - obligé - pour que le dissensus exacerbé des grillini circule dans le corps social, en marquant à la fois le passage à la manifestation discursive et à la notoriété médiatique. Et c'est à partir de ce moment, en passant par le Cozza Day de Rome31', le 11 septembre 2012, et jusqu'au troisième Vaffa Day de Gênes, le 3 décembre 2013, que la formule enregistre son expansion maximale. De la place virtuelle du Web à la place publique et aux médias, on assiste à l'émergence d'une ronde de réénonciations dont la formule Vaffa Day constitue, en tant que matrice, l'épicentre. Réénonciations qui, «du fait de leurs emplois à un moment donné et dans un espace public donné, cristallisent des enjeux politiques et sociaux» (KriegPlanque, 2003, p.7), et qui retravaillent la formule primaire en la projetant sur deux axes isotopiques : le premier, qui passe par la sloganisation; le deuxième, qui joue sur l'estropiement du nom propre. Il s'agit, dans les deux cas, de réorienter le vaffa dans une chaîne de résonances misant, en l'absence d'une véritable argumentation, sur les valeurs affectivo-instinctuelles, et se développant sur le fil d'une arborescence de connexions s'attirant entre elles dans un système de désignations sémantiquement - et axiologiquement - liées.

\section{Sloganisation et estropiement du nom propre}

À la suite de Maurice Tournier, nous entendons par sloganisation « tout usage de répétitivité formelle et de liaisons récurrentes dans les énoncés propres à l'intervention publique». Cet usage, continue Tournier, repose sur «certaines habitudes d'attirance que les mots entretiennent les uns avec les autres », en remodelant «la "pâte" dont un texte est confectionné » selon une récurrence qui «peut aller de monotones séquences graphiques quasi figées à une fluidité d'énoncés hétérogènes à forme variée » (Tournier, 1985, note 11, p. 158). Ce qui fait de la sloganisation ce mécanisme à travers lequel le discours s'insère "dans un tissu de redondances organisées " $3^{2}$, censées restituer dans un effet de martèlement une série de cooccurrences se rattachant à un pôle primaire.

31. Cozza day, c'est-à-dire «la journée de la moule », pour protester contre les parlementaires s'accrochant au pouvoir à la façon des mollusques s'accrochant aux rochers.

32. Comme ne manque pas de le rappeler, en citant Tournier, le Dictionnaire d'analyse du discours à l'entrée «sloganisation» (Charaudeau, Maingueneau, 2002, p. 537). 
Et c'est exactement ce qui se produit dans notre cas : le syntagme Vaffa Day, pôle primaire et organisateur, donne libre cours à une «fluidité d'énoncés» rendant compte dans le tissu du discours du degré d'attirance qu'ils exercent «à longue portée »33, au-delà de l'espace immédiat de la formule. Nous avons retenu «Tutti a casa» («Tous à la maison»), «Fuori tutti» («Tous dehors»), «Licenziamoli tutti» ("Licencions-les tous»), trois énoncés cooccurrents, qui reformulent dans un rapport d'attirance réciproque l'exhortation indignée à ficher le camp, intrinsèque à la formule-matrice Vaffa Day. Qu'il nous suffise de donner quelques exemples tirés du blog de Grillo :

E' NATO TUTTO CON UN VAFFANCULO URLATO...

HAAAAA !!! CHE SENSO DI LIBERAZIONE!!!!

UN VAFFANCULO URLATO DOVREBBE ACCOMUNARE RAZZE, RELIGIONI, IDEOLOGIE, SESSI (magari!).

un vaffanculo urlato... tanto per cominciare... e per distinguersi...

Quando il «potere» incombe... solo il concetto poetico di anarchia si erge a suo baluardo.

[...]

TUTTI A CASA E TUTTI A FANCULO!!!

Paolo C. (out of the blue) (17 avril 2008, 12 h 58)34

Fuori tutti. In quindici anni avete riportato il Paese al dopoguerra. Sono in milioni, in Italia, a pensarla così su chi oggi governa la nazione.

GRANDE GRILLO FUORI TUTTI

Massimo Scifo (Gênes, 8 mars 2008, 9 h 55) 35

Licenziamoli tutti questi impasticciati dilettanti furbacchioni, sono solo dei paraculi e del popolo non gliene frega niente!

Franco M. (23 septembre 2007,18 h 55) 36

Le passage du blog à la presse permet de constater que le même principe d'attirance organise la texture de plusieurs titres :

- «Beppe Grillo n'est pas Coluche» (rue8glyon.fr, 8 février 2013);

33. En analysant les tableaux où sont reportées les liaisons statistiques d'un faisceau de corrélations présent dans des textes de propagande, Tournier écrit : «Or, les tableaux initiaux nous montrent que les attirances font sentir leurs effets non seulement dans l'espace immédiat du syntagme mais également à longue portée, jusqu'au fond des phrases.» (Tournier, 1985, p.171-172)

34. «Tout a commencé par un va-te-faire-foutre hurlé... / ah, quelle sensation de liberté !!!! / un vate-faire-foutre hurlé devrait rapprocher les races, les religions, les idéologies, les sexes (carrément!). / Un va-te-faire-foutre hurlé... ce n'est que le début... et pour se distinguer... / Quand le «pouvoir» nous menace, seule la notion poétique d'anarchie peut nous servir de rempart. [...] Tous à la maison et allez tous vous faire foutre! » Voir http://www.beppegrillo.it/2008/04/ domani.html.

35. «Tous dehors. Pendant ces quinze dernières années vous avez ramené le pays à l'après-guerre. C'est l'opinion de millions d'Italiens aujourd'hui à propos de notre classe gouvernante. / Grillo, t'es grand! tous dehors! „Voir : http://www.beppegrillo.it/2008/03/fuori_tutti.html.

36. "Licencions-les tous ces embrouilleurs, ces politicailleurs de quatre sous, ces filous, ils ne sont que des branleurs et ils s'en fichent du peuple !» Voir : http://www.beppegrillo.it/2007/09/ ammazzateci_tu.html. 
- «Beppe Grillo trublion de la politique italienne » franceinfo.fr, 23 février 2013);

- «Beppe Grillo : “Abbiamo fatto fuori 5 partiti. Tutti a casa...” » (L'Indipendenza.com, 20 avril 2013);

- «Grillo : "Tutti a casa, anche noi” » (Espresso.it, 26 août 2013);

- «Intervista a Beppe Grillo con Metro : “Tutti a casa. L'Italia come l'Egitto, e io sono il garante della rivoluzione" " (Huffinghton Post, 10 avril 2011);

- "Grillo parla con Metro : "tutti a casa, questi qui” » (Metro World News, 10 avril 2013).

Quant au deuxième axe isotopique, celui de l'estropiement du nom propre, il s'agit d'une pratique qui répond ad personam, sous forme d'attaque frontale directe, à la rhétorique du vaffa, en lui conférant une caractérisation nominale et en sollicitant à la fois l'argument ad populum. "Lorsque l'on considère l'argument ad populum », remarque Ruth Amossy en citant Walton, « on a tendance à y voir un appel à la foule où la passion rhétorique cherche à mobiliser le peuple en vue d'une action portée par l'enthousiasme, voire en vue d'un déferlement de violence» (Amossy, 2006, p. 184). Or, dans notre cas, il est intéressant de noter que le travestissement du nom propre et le sobriquet ridicule, outre qu'ils constituent, comme le soulignent bien Paola Paissa et Ruggero Druetta (2014), « une stratégie systématique » d'attaque des adversaires, « utilisée à toute époque, de préférence par les partis conservateurs et les mouvements populistes », constituent aussi pour Grillo une pratique privilégiée pour faire appel à la foule et susciter un effet de « ras-le-bol». Mieuxvaudrait dire un effet «tsunami »37, en vue d'un « déferlement» où l'émotif déborde le rationnel et recoupe, d'une façon presque torrentielle, le langage de la violence et du discrédit. On assiste ainsi à une cascade de désignations « qui sont avant tout des prises de position dans le champ politique et dans le champ de bataille du discours» (Siblot, 2007, p. 38). Ces redésignations appliquent des étiquettes qui stigmatisent, en les ridiculisant, les antagonistes, de sorte que Berlusconi devient le «psiconano» [psychonain]; Mario Monti, «Rigor Montis», calembour soudant l'austérité à la rigidité cadavérique; Bersani, «zombie», et occasionnellement «Gargamella » [Gargamel], le sorcier spécialisé dans l'alchimie; Giorgio Napolitano, «cadavere» [cadavre] ou «boia » [bourreau]; Enrico Letta, "Capitan Findus Letta», leader du "surgelé»; le parti de gauche, soit le PD, «pdmenoelle» [pd-moins-elle], pour en souligner l'« inciucio »38 avec le PDL; Matteo Renzi, «Fonzie», en raison de sa veste en cuir noir; Walter Veltroni, la

37. Il s'agit là d’une allusion voulue au «Tsunami tour», la tournée électorale qui, au lendemain du premier Vaffa Day, emporte Grillo du nord au sud de l'Italie à bord d'un camping-car. Le syntagme, dès son apparition, se met à circuler dans l'univers des discours médiatiques et, très rapidement, accède au statut de formule. Les réénonciations et les emplois de cette formule, ainsi que ses relations avec la formule Vaffa Day, mériteraient une étude à part.

38. Terme désignant de manière péjorative un accord préélectoral entre les partis de la majorité et ceux de l'opposition. 
petite souris «Topo Gigio »... Elles font partie d'un inventaire qu'on pourrait dresser à l'infini, dessinant un répertoire dysphorique d'appellations à forte charge émotionnelle, qui l'emportent largement sur les jeux de mots qu'elles charrient apparemment, en déterminant ce qu'Algirdas Greimas et Jacques Fontanille appellent «l'“irruption” du somatique à la surface du discours » et dont l'apparition «signale que la jonction thymique est accomplie, en donnant la parole au corps propre» (Greimas, Fontanille, 1991, p.170). Ces désignations, encore, convoquent le sujet "sentant», qui s'inscrit dans la chaîne discursive selon les modulations du «faire pathémique». Et c'est justement dans ce schéma pathémique que Grillo brandit l'arme de la dérision du nom propre : les noms estropiés font irruption dans ses discours en guise de sanction du «corps » de l'adversaire, devenant assez rapidement une référence obligée du blog et des médias, où ils circulent en stricte association avec l'idée d'une politique rénovatrice que la formule Vaffa Day prétend résumer.

Mais ce n'est pas tout. Car la formule Vaffa Day enregistre une extension supplémentaire au moment de la présentation du logo en vue des élections municipales de 2008, et des élections législatives de 2013. Elle est réénoncée par le biais d'un défigement iconique qui en exploite le potentiel intersémiologique : on écrit Lista CiVica et MoVimento 5 Stelle avec un V majuscule de couleur rouge, astuce graphique censée faire résonner le vaffa sous-jacent, en imprégnant le logo de la même verve caustique qui alimente le Vaffa Day. Le pouvoir évocateur de ce $V$ majuscule est tel qu'on commence à parler de $V$ generation. Se dessine ainsi un parcours où le comportement de la formule Vaffa Day se laisse appréhender comme la propagation, sur sa propre trajectoire, d'une sorte d'arborescence dont elle constitue la manifestation primaire. On comprend alors, rétrospectivement, pourquoi nous avons voulu la dénommer formule filée.

\section{De la formule à l’idiome : le grillese}

Évoquer à propos du Vaffa Day une formule filée nous permet d'envisager dans l'espace de son rayonnement une perspective complémentaire portant sur sa productivité néologique. On rencontre ainsi un triplet de synonymes qui lui sont directement tributaires et font partie d'un clavier verbal relevant d'une langue grossière, corporellement "contaminée», se manifestant par des corrélats morphologiques et métonymiques, soit : fanculare, sfanculare, sfanculizzare. Mots-virus, vénéneux, qui investissent l'autre - l'adversaire - en lui inoculant une sorte de poison rhétorique visant à le réduire au silence 39 ; mots

39. Ce que Casaleggio dit de Grillo et du M5S lors d'une interview dans The Guardian le 3 janvier 2013 est à cet égard significatif : "It's like Jesus Christ and the apostles. His message, too, became a virus. » Alors que Lucy Geffroy, au lendemain de la sortie du livre /l Grillo canta sempre al tramonto, écrit dans Le Monde diplomatique de septembre 2013: «Le M5S est défini par Casaleg- 
se caractérisant par leur volonté d'annihiler l'adversaire en lui ôtant a priori toute possibilité de confrontation, délégitimant sa «face» au nom de ce qu'il a de plus ignoble - son «cul». Les forums en ligne deviennent ainsi l'arène où regorge une violence verbale hors de l'ordinaire. On assiste à l'éclosion d'un langage trivial, contagieux, qui fait appel au «bas», à la vulgarité, et qui se définit par un schéma postural de nature viscérale, comme en témoigne ce message posté à la veille du troisième Vaffa Day: "Beppe Grazie, mi hai messo il cervello nella pancia. $\gg 40$

«Le cerveau dans le ventre»: c’est l'archéologie du grillese, idiome fédérant dans une nouvelle forme de populisme digital les câbles des ordinateurs et qui répond, d'après Giuliano Santoro, à la frustration émotionnelle des Italiens déçus par les technocrates de la politique. Le grillese représente, dans ce sens, l'intensité d'un sentiment d'incompréhension qui inscrit dans les déclinaisons « corporelles » du vaffa le tonus d'une colère donnant en spectacle grotesque la promesse d'un avenir meilleur. En témoigne, entre autres exemples, l'exorde de la lettre que Grillo écrit le 24 décembre 2008 au Père Noël :

Caro Babbo Natale,

Nel 2008 non sono stato buono, anche quest'anno ho mandato a fanculo tanta gente. Ho trattato male i giornalisti, gli editori, i politici, gli imprenditori con le pezze al culo. Portami lo stesso qualcosa. 41

Et ses blogueurs de faire écho :

BUON NATALE E BUON FANCULO YEAR A TUTTI.

Roberto S. (Palerme, 25 décembre 2012, 12 h 28)42

caro babbo natale, vorrei che una meteora si abbattesse su roma! tu mi dirai ma sei matto perdere un patrimonio come roma, il colosseo, la fontana di trevi, piazza navona, tutta roma antica. Non me ne frega un cazzo! [...]

con affetto tuo luigino

Luigi Verruti (fanculosilvio) (Cuneo, 25 décembre 2008, 20 h 11)43

Il est ici intéressant de constater l'assimilation identitaire que le grillese produit là où le blogueur accole à son nom le pseudonyme fanculosilvio.

gio comme l'“amanite phalloïde” des partis - un champignon vénéneux connu pour avoir provoqué la mort de figures historiques telles que l'empereur romain Claude.»

40. "Merci Beppe, tu m'as mis le cerveau dans le ventre. " (Roberto Ghidoni, Andora, 2 décembre 2013, 10 h 46) : http://www.beppegrillo.it/2013/12/la_modesta_proposta_di_beppe_grillo_ al_v3day_-_oltre.html.

41. "Cher Père Noël, / En 2008 je me suis mal conduit, même cette année-ci j’ai envoyé se faire foutre beaucoup de gens. J'ai mal traité les journalistes, les éditeurs, les politiciens, les entrepreneurs dans la merde jusqu'au cou. Apporte-moi quelque chose quand même. » http://www. beppegrillo.it/2008/12/lettera_a_babbo.html.

42. "Joyeux Noël et joyeux fanculo year à tous.»

43. "Cher Père Noël, je voudrais qu'une météorite tombe sur Rome! On me dira : mais tu es fou, perdre un patrimoine artistique comme le Colysée, la fontaine de Trevi, Piazza Navona et la Rome antique. Je m'en fous ! [...] / Avec affection, ton ami Luigino.» 
Derrière le recours au grillese en tant que construction d'un idiome, on retrouve le grillese en tant que construction d'un masque, c'est-à-dire une articulation emphatique qui traduit, pour le dire avec Bakhtine écrivant sur Rabelais, «la joyeuse négation de l'identité et du sens unique »44. Tout se joue dans le cadre d'une sorte de théâtralité qui se révèle incorporée à la nature même du grillese, comme l'a d'ailleurs bien expliqué le journaliste Filippo Ceccarelli :

Le «grillese» mériterait nombre d'investigations scientifiques. En l'écoutant, tu y entends la «commedia dell'arte», l'incessant bruissement ligurien, la culture publicitaire et télévisuelle, beaucoup de Fantozzi, un brin de Pannella (ce n'est pas un hasard s'il a accusé Grillo de le copier), un peu de Dagospia, des échos des chats et, de même, des résonances futuristes rappelant D’Annunzio [...] Mais le voir c'est encore mieux, parce qu'il a du tempo, du rythme, il fait des grimaces irrésistibles, comme il arrive quand il imite Bossi et sa bouche tordue...45

Le grillese, autrement dit, est à l'image et à la ressemblance de sa grande gueule : Beppe Grillo, le «roi bouffon» et barbu, le «clown hurlant» qui parle aux foules «avec ses tripes " 46 ; ou encore, le «messie» au verbe et au geste prophétiques47, qui galvanise le Web au nom d'une émotion et d'une rédemption virtuelles.

Dans une lettre de Quintus à Cicéron au début de l'année 64 av. J.-C., au moment où son illustre frère aîné entre en lice pour les élections consulaires, il lui adresse des conseils pratiques qui radiographient dans les moindres détails le fonctionnement de la machine électorale. La préoccupation majeure est celle de s'assurer le «consensus omnium», et à cette fin Quintus esquisse un programme où, en dressant une sorte de vademecum tactique, il recommande à Cicéron des démarches stratégiques minutieusement décrites. En particulier: la répartition topographique, soit « une vue d'ensemble de toute l'Italie répartie par tribus, afin de ne laisser aucun municipe, aucune colonie, aucune préfecture, enfin aucun coin de l'Italie où tu n'aies ce qu'il faut d'appui» (Prost, 2009, p. 32) ; la faveur populaire, qui requiert « d'être constamment présent, de faire preuve de générosité, de faire parler de soi, de faire naître des espérances politiques» (ibid., p. 38) ; la présence constante à Rome, car «à être constamment présent on gagne non seulement d'être toujours à Rome et sur le forum, mais de faire campagne constamment, de s'adresser souvent aux mêmes personnes, et d'éviter que quiconque (autant que possible) puisse dire qu'il n'a

44. Et encore Bakhtine (1970, p. 49) : «Il faut simplement noter que des phénomènes comme la parodie, la caricature, la grimace, les simagrées, les singeries, ne sont au fond que des dérivés du masque.»

45. F. Ceccarelli, «Il linguaggio dell'esasperazione», LaRepubblica.it, 5 septembre 2012.

46. Comme l'écrit P. de Gasquet, «Pier Luigi Bersani : l'ultime rempart? ", LesEchos.fr, 6 mars 2013.

47. C'est Casaleggio qui compare Grillo à Jésus lors de son interview-choc dans The Guardian le 3 janvier 2013. 
pas été sollicité par toi, et sollicité avec insistance et application » (ibid., p. 39). Pour que la campagne soit gagnante, ajoute Quintus, elle doit être «pleine de pompe, brillante, splendide et populaire» et doit aller de pair avec le discrédit de l'adversaire : «si possible de quelque manière, diffuse concernant tes concurrents une rumeur infamante de crime, d'immoralité ou de corruption accordée à leurs mœurs » (ibid., p. 43-44). Un rôle décisif en vue de la victoire est aussi accordé au langage, plus précisément un langage « qui convient à ce dessein », qui permet de se garantir le soutien le plus large possible et qui soit à même d'arriver directement à la masse en tissant des liens personnels, « de sorte qu'on dise et pense que tu connais bien tes concitoyens» (ibid., p.42).

Dans le cadre de notre analyse, la lettre de Quintus à Cicéron fournit, à distance de deux millénaires et à l'ère du Web 2.0, des éléments précieux de réflexion, pour aller au cœur des stratégies d'ascension politique de Grillo et du M5S. Mutatis mutandis, le blog et les réseaux sociaux, tout particulièrement les MeetUp, permettent une extension capillaire du «forum » en tant qu'espace de discussion en Italie et dans le monde : des grandes villes aux endroits les plus reculés, de Rome à Paris, de Londres à San Francisco, Grillo est «constamment présent», il fait appel à la foule en sollicitant ses concitoyens «avec insistance et application», il fait "parler de lui », il instille des «espérances politiques». Dans un éternel présent $4^{8}$, son blog recouvre dans une "vue d'ensemble» le temps et l'espace, il rassemble la masse dans une émotion toute virtuelle en polarisant le langage du côté du mal-être, du côté d'une « rumeur infamante de crime, d'immoralité ou de corruption», en démontrant que l'adversaire entretient avec le peuple un rapport fait de rouerie et tromperie. Dans ce cadre, la formule Vaffa Day circule en corrélation «à des énoncés qui la soutiennent, la portent, la reprennent, la renforcent, la réitèrent ou la récusent» (Krieg-Planque, 2003, p.73), sa concision lui permet de s'affirmer dans la matérialité linguistique du discours et de remplir un vide émotionnel où l'ironie, la caricature, la grimace et les jurons donnent libre cours à la passion en termes de massification des émotions. Sorte de formule biblique renversée, que plusieurs, en parlant de "fascisme numérique», ont rapprochée du «Me ne frego» [je m'en fous] de Mussolini et des «squadristi»49, elle se révèle orientée, pour le dire encore avec Krieg-Planque, «par la morphosyntaxe et les composantes lexicales de la séquence» (Krieg-Planque, 2009, p. 108), et se propage dans l'espace public en exploitant, d'une part, la "polimicité» du signifiant, et en s'adressant, d'autre part, à une multitude indistincte où

48. «Dans le monde de Beppe Grillo, le passé n’existe pas. Il existe seulement le futur de la “nouvelle ère", l'ère du Réseau, de la mutation anthropologique. » (Santoro, 2012, p. 116).

49. Parmi les nombreux articles, nous nous bornons à citer celui de R. Ronza : «Quando i grillini fanno rima con Mussolini », ainsi que la réflexion de F. Mulongo : «Beppe Grillo les a tous grillés!», respectivement consultables sur les sites http://www.lanuovabq.it/it/articoliquando-i-grillini-fanno-rimacon-mussolini-7901.htm et http://www.reveil-fm.com/index. php/2013/02/28/3283-italie-beppe-grillo-les-a-tous-grilles. 
l'individu est considéré comme faisant partie d'un essaim sans identité et sans visage. Car derrière le vaffa et la manière de nommer inscrite dans ses tournures polémiques, il n'y a aucune visée argumentative, il n'y a ni un opposant ni un tiers, mais uniquement un format et un visage : Beppe Grillo.

La moralité de cette fable grotesque est alors évidente : il s'agit de tirer la langue, la portant «dans l'autre sens ॥50, à l'extérieur, au sens d'une «traction» qui la fait «avancer hors de la bouche», en dehors de toute intention proprement communicative. Une langue qui ne dit plus, mais qui tranche net la parole en tant que logos, et qui vise à «solidifier en grimaces durables» sa grâce ${ }^{51}$, en faisant appel au destinataire et à ses passions pour l'amuser et le réduire au silence.

\section{Références}

Amossy Ruth, 2006, l'argumentation dans le discours, Paris, Armand Colin.

BAKHTINE Mikhaïl, 1970, L'œuvre de François Rabelais, Paris, Gallimard.

Bergson Henri, 2002 [1900], Le Rire. Essai sur la signification du comique, Paris, PUF [Alcan], http://www.ebooksgratuits.com/html/bergson_le_rire.html.

BRANTS Kees, 2003, «De l'art de rendre la politique impopulaire... Ou qui a peur de l'infotainment?», Réseaux, nº 118, p. 135-166.

CASALEggIo Gianroberto, 2001, /l Web è morto, viva il Web, s.l., Pro-Sources.

- 2004, Web ergo sum, Milan, Sperling \& Kupfer.

Charaudeau Patrick, Maingueneau Dominique, 2002, Dictionnaire d'analyse du discours, Paris, Le Seuil.

Duвoıs Philippe, 1975, «La métaphore filée et le fonctionnement du texte », Le français moderne, $\mathrm{n}^{\circ} 43$ (3), juillet, p. 202-213.

GreimAS Algirdas Julien, FonTANILLE Jacques, 1991, Sémiotique des passions. Des états des choses aux états d'âme, Paris, Le Seuil.

GrILlo Beppe, 2006, Tutto il Grillo che conta, Rome, Feltrinelli.

Kerbrat-ORECCHIONI Catherine, 1980, Le discours polémique, Lyon, Presses universitaires de Lyon.

KRIEG-PLANQUE Alice, 2003, "Purification ethnique ». Une formule et son histoire, Paris, CNRS.

- [Entretien avec], 2006, “"Formules” et “lieux discursifs”. Propositions pour l'analyse du discours politique, Semen, n²1, 2006, en ligne : http://semen.revues.org/1938.

- 2009, La notion de "formule » en analyse du discours. Cadre théorique et méthodologique, Besançon, Presses universitaires de Franche-Comté.

50. Voir l'entrée «tirer» du Trésor de la langue française, http://atilf.atilf.fr.

51. Nous faisons ici allusion à la définition du «comique» que donne H. Bergson, et tout particulièrement dans le passage suivant : «Là où la matière réussit ainsi à épaissir extérieurement la vie de l'âme, à en figer le mouvement, à en contrarier la grâce, elle obtient du corps un effet comique. Si donc on voulait définir ici le comique en le rapprochant de son contraire, il faudrait l'opposer à la grâce plus encore qu'à la beauté. » (Bergson, 2002 [1900]). 
Mello Federico, 2013, Il lato oscuro delle stelle, Milan, Feltrinelli.

MoIRAND Sophie, 2007, Les discours de la presse quotidienne. Observer, analyser, comprendre, Paris, PUF.

Oustinoff Michaël, 2010, «Langues de bois d'hier et parler vrai d'aujourd'hui. De la "novlangue" aux "spin doctors" », Hermès, no 58 , 2010, p. 15-21.

PAISSA Paola, DruetTA Ruggero, 2013, «De Berlusconi à... berlusconiesque : polymorphies du nom propre dans le discours médiatique français», Linguistica applicata con stile. In traccia di Bice Mortara Garavelli, F. Geymonat éd., Alessandria, Edizioni dell'Orso, p.105-127.

Plantin Christian, 1996, L'argumentation, Le Seuil, Paris.

— 2003, "Des polémistes aux polémiqueurs», La parole polémique, G. Declercq, M. Murat, J. Dangel éd., Paris, Champion, p. 277-308.

PREgliAsCo Lorenzo, 2013, Il crollo. Dizionario semiserio delle 101 parole che hanno fatto e disfatto la Seconda Repubblica, Rome, Editori Internazionali Riuniti.

PROST François, 2009, Quintus Cicéron. Le petit manuel de la campagne électorale (Commentariolum petitionis), Tulliana, en ligne : http://www.tulliana.eu/documenti/BindercommentariolumProst.pdf

RiffaterRe Michel, 1979, "La métaphore filée dans la poésie surréaliste », La production du texte, Paris, Le Seuil.

SANToro Giuliano, 2012, Un Grillo qualunque, Rome, Castelvecchi.

Sib Lot Paul, 2007, Nomination et point de vue. La composante déictique des catégorisations lexicales, dans L'acte de nommer. Une dynamique entre langue et discours, G. Cislaru et al., Paris, Presses de la Sorbonne nouvelle.

TOURNIER Maurice, 1985, "Texte propagandiste et cooccurrences. Hypothèses et méthodes pour l'étude de la sloganisation », Mots, octobre, nº 11, p. 155-187. 
